\title{
The nexus between energy price changes and environmental quality in Malaysia
}

\begin{abstract}
This paper provides new evidence by examining the impact of oil price changes on environmental quality. Having utilized three co-integrating estimators and a dataset from 1983 to 2014, we found that lower oil price can increase carbon emissions and reduce environmental quality. On the other hand, higher oil price can mitigate carbon emissions and improve environmental quality. We recommend the use of cleaner energy sources to improve environmental quality during lower oil price.
\end{abstract}

Keyword: Oil price; Environmental quality; Malaysia 